\title{
Perphenazine exerts antitumor effects on HUT78 cells through Akt dephosphorylation by protein phosphatase $2 \mathrm{~A}$
}

\author{
SHUNYA TSUII $^{1}$, NAOKI KOHYANAGI ${ }^{1}$, TAKUYA MIZUNO ${ }^{2}$, TAKASHI OHAMA ${ }^{1}$ and KOICHI SATO ${ }^{1}$ \\ ${ }^{1}$ Laboratory of Veterinary Pharmacology and ${ }^{2}$ The Laboratory of Molecular Diagnostics and Therapeutics, \\ Joint Faculty of Veterinary Medicine, Yamaguchi University, Yamaguchi 753-8515, Japan
}

Received May 30, 2020; Accepted September 9, 2020

DOI: $10.3892 / \mathrm{ol} .2020 .12374$

\begin{abstract}
Sezary syndrome is a rare type of non-Hodgkin lymphoma. Protein phosphatase 2A (PP2A) is an important tumor suppressor whose activity is widely inhibited in a variety of tumors. Recently, reactivation of PP2A has attracted increasing attention as a promising approach for cancer therapy. Phenothiazine anti-psychotic perphenazine (PPZ) exerts antitumor effects by reactivating PP2A. The present study investigated the molecular mechanism underling the antitumor effects of PPZ in the neuroblastoma rat sarcoma oncogene (NRAS)-mutated Sezary syndrome cell line, HUT78. The results of the present study demonstrated that PPZ induced the dephosphorylation of Akt and ERK1/2, and triggered apoptosis in HUT78 cells. In addition, a PP2A inhibitor blocked the PPZ-mediated dephosphorylation of Akt but did not affect that of ERK1/2. The pharmacological inhibition of Akt and ERK1/2 signaling revealed that Akt activity serves an important role in the survival of HUT78 cells. The present data suggested that suppressing Akt activity by PP2A activation may be an attractive antitumor strategy for NRAS-mutated Sezary syndrome.
\end{abstract}

\section{Introduction}

Sezary syndrome is a rare type of non-Hodgkin lymphoma, a common form of cutaneous T-cell lymphomas (1). Although the pathophysiology of Sezary syndrome is not completely

Correspondence to: Professor Takashi Ohama, Laboratory of Veterinary Pharmacology, Joint Faculty of Veterinary Medicine, Yamaguchi University, 1677-1 Yoshida, Yamaguchi 753-8515, Japan E-mail: t.ohama@yamaguchi-u.ac.jp

Abbreviations: CsA, cyclosporin A; NRAS, neuroblastoma rat sarcoma oncogene; OA, okadaic acid; PI, propidium iodide; PI3K, phosphoinositide 3-kinase; PP2A, protein phosphatase 2A; PPZ, perphenazine; SDS, sodium dodecyl sulfate; T-ALL, T-cell acute lymphoblastic leukemia; Tau, tautomycin; VCP, valosin-containing protein

Key words: Akt, HUT78, perphenazine, protein phosphatase 2A, Sezary syndrome understood, it has been reported that a $R A S$ gene mutation is associated with its poor prognosis (2). HUT78 is a Sezary syndrome cell line, containing the neuroblastoma rat sarcoma oncogene (NRAS) ${ }^{\mathrm{Q} 61 \mathrm{~K}}$ mutation and an amplified RAS/RAF/MEK signaling pathway (2). This mutation sensitizes the HUT78 cells toward suppression of the RAS/RAF/MEK signaling pathway by NRAS knockdown as well as treatment with MEK inhibitors (2). Phosphoinositide 3-kinase (PI3K)/Akt signaling is the second best validated NRAS downstream (3), and melanoma cell lines harboring NRAS ${ }^{\mathrm{Q} 61 \mathrm{~K}}$ mutation are sensitive to the combination of MEK/ERK and PI3K/Akt signaling inhibitors (4). However, HUT78 cells are refractory to the PI3K inhibitors, maybe because of the existence of PTEN (5), suggesting PI3K-independent suppression of Akt activity is necessary.

Kinase inhibitors are indispensable in the current therapy field for diseases with protein hyperphosphorylation, especially cancer. However, there are various problems associated with these inhibitors, such as drug resistance and limited targets. Recently, reactivation of protein phosphatase 2A (PP2A) has attracted a lot of attention as a different angle to block protein hyperphosphorylation (6). PP2A is a highly conserved eukaryotic serine/threonine protein phosphatase that regulates a wide range of intracellular signal transduction pathways, including MEK/ERK and PI3K/Akt (7-9). In T-cell acute lymphoblastic leukemia (T-ALL) and chronic lymphocytic leukemia, PP2A activity is low, and its reactivation exerts antitumor effects $(10,11)$. Perphenazine (PPZ) is a classically used phenothiazine in neuroleptic-type anti-psychotic medications (12). Previous research, including our own, showed that PPZ exerts antitumor effects in T-ALL cells through PP2A activation $(10,13)$. Because Akt is a substrate of PP2A, PP2A activation is expected to directly inhibit Akt activity and induce apoptosis in cancer cells that are resistant to PI3K inhibitors. However, it is unknown whether PP2A reactivation by PPZ also exerts antitumor effects on HUT78.

In this study, we revealed that PPZ exerts antitumor effects on HUT78 by dephosphorylating Akt in a PP2A-dependent manner. Interestingly, PPZ also dephosphorylated ERK1/2; however, PP2A was not involved in this process, and an ERK1/2 inhibitor did not suppress cell growth. In HUT78 cells, MEK inhibition led to dephosphorylation of Akt, resulting in antitumor effects. Our findings suggest that Akt inhibition by PP2A re-activation may be an effective strategy for the treatment of NRAS-mutated Sezary syndrome. 


\section{Materials and methods}

Reagent and antibodies. The reagents used in this study were as follows: PPZ and FR180204 (Sigma-Aldrich), Akt inhibitor VIII (Santa Cruz Biotechnologies.), BCI (Merck Millipore), and U0126 (LC Laboratories.). The antibodies used in this study were as follows: Anti-p97/VCP, anti-B-Raf, anti-N-Myc downstream-regulated gene 1 (NDRG1) (GeneTex), anti-cleaved caspase 3 Asp175, anti-pThr202/Tyr204 ERK1/2, anti-pThr308 Akt, anti-pSer473 Akt, anti-pSer241 PDK1, anti-PDK1, anti-pThr346 (NDRG1), anti-pSer217/221 MEK1/2, anti-pSer445 B-Raf, anti-pSer338 C-Raf, anti-ERK1/2, and anti-Akt (Cell Signaling), anti-C-Raf (BD Biosciences).

Cell culture. Human T cell acute lymphoblastic leukemia cell lines (CCRF-CEM, Jurkat, TALL1) and the human Sezary syndrome cell line (HUT78) were obtained from RIKEN BRC. The canine $\mathrm{T}$ cell acute lymphoblastic leukemia cell line (UL-1) was kindly provided by Dr Hajime Tsujimoto. MycoAlert Mycoplasma Detection kit (Lonza) was used to test mycoplasma contamination. The cells were cultured in RPMI1640 (Sigma-Aldrich) containing 10\% fetal bovine serum (FBS, Nichirei Biosciences) and antibiotic/antimycotic (Nacalai Tesque).

Cell viability assay. Cells $\left(4.0 \times 10^{4}\right)$ were seeded on 96-well plates, and drugs were added to the medium after $24 \mathrm{~h}$. Following three-day culture, cell viability assays were performed using Cell Counting Kit-8 (CCK8, Dojindo) as previously described (13).

Flow cytometry. HUT78 cells were treated with PPZ $(20 \mu \mathrm{M})$ for $12 \mathrm{~h}$. Apoptotic cells were stained with Annexin V-FITC Apoptosis Detection Kit (Bio Vision) as per the manufacture's instructions. Annexin V-and propidium iodide-positive cells were detected by flow cytometer (BD Accuri; BD Biosciences) as previously described (13).

Immunoblotting. Immunoblotting was performed as previously described (13). Briefly, cell lysis buffer containing $50 \mathrm{mM}$ Tris- $\mathrm{HCl}$ ( $\mathrm{pH}$ 8.0), $5 \mathrm{mM}$ ethylenediaminetetraacetic acid (EDTA) (pH 8.0), $5 \mathrm{mM}$ ethylene glycol tetraacetic acid (EGTA), $1 \%$ Triton $\mathrm{X}-100,1 \mathrm{mM} \mathrm{Na} \mathrm{VO}_{4}, 20 \mathrm{mM}$ sodium pyrophosphate and Roche's complete protease inhibitor cocktail. The protein concentrations were measured by DC protein assay kit (Bio-Rad Laboratories, Inc.), and were separated by SDS-PAGE, then transferred onto ClearTrans Nitrocellulose Membrane (Wako). Membranes were blocked with 0.5 or $3 \%$ skim milk, and treated with primary antibodies in Tris-buffered saline (TBS) containing 0.05\% Tween-20 (TBS-T). After treating with secondary antibodies in TBS-T, immunoreactive bands were detected using ECL Pro Western Blotting Detection Reagent (PerkinElmer.) and visualized using a LAS-3000 luminescent image analyzer (Fujifilm) or ImageQuant 800 (GE Healthcare). Valosin-containing protein (VCP) was used as a loading control.

In vitro kinase assay. Active-MEK1 (50 ng) (SignalChem, M02-10G) and $200 \mathrm{ng}$ of unactive-ERK1 (SignalChem, M29-14U) were mixed into kinase buffer (25 mM MOPS
$\mathrm{pH} 7.2,12.5 \mathrm{mM}$ sodium glycerophosphate, $25 \mathrm{mM} \mathrm{MgCl}_{2}$, $5 \mathrm{mM}$ EGTA, $2 \mathrm{mM}$ EDTA, and $0.25 \mathrm{mM}$ DTT) with or without PPZ $(10 \mu \mathrm{M})$, and rotated for $1 \mathrm{~h}$ at $4^{\circ} \mathrm{C}$. Thereafter, $25 \mathrm{mM}$ of ATP were added and incubated for $15 \mathrm{~min}$ at $30^{\circ} \mathrm{C}$. The reaction was stopped by adding sodium dodecyl sulfate (SDS) sample buffer and samples were incubated for 5 min at $100^{\circ} \mathrm{C}$. Total ERK1 and ERK1 phosphorylation were detected by immunoblotting.

Statistical analysis. The results are expressed as means \pm standard deviation. Student's t-tests were performed to compare two groups. Comparison of three or more was performed using one-way analysis of variance (ANOVA), followed by Fisher least significant difference test. $\mathrm{P}<0.05$ was considered to indicate a statistically significant difference.

\section{Results}

PPZ induces apoptosis in HUT78 cells. We investigated whether PPZ exerts antitumor effects in Sezary syndrome cells as well as in T-ALL cells. In addition, we also compared the sensitivity of the NRAS-mutated Sezary syndrome cell line (HUT78) to PPZ with that of T-ALL cell lines (CCRF-CEM, Jurkat, and TALL1). PPZ suppressed the viability of HUT78 cells in a dose-dependent manner, and the same was observed in T-ALL cell lines (Fig. 1A). Because PP2A reactivation has been reported to induce apoptosis in cancer cells $(10,14)$, we investigated whether PPZ also induces apoptosis in HUT78 cells. Immunoblotting results revealed that PPZ increased the expression of cleaved (active) caspase 3 in HUT78 cells (Fig. 1B and C). Moreover, flow cytometry analysis showed that PPZ treatment resulted in an increased number of early-phase apoptotic cells, shown as Annexin V-positive and propidium iodide (PI)-negative, as well as Annexin V and PI positive late-phase apoptotic/necrotic cells (Fig. 1D and E). These results suggest that PPZ induces apoptosis in HUT78 cells.

PPZ induces dephosphorylation of PP2A substrates. In T-ALL cells, PPZ induces dephosphorylation of PP2A substrates, such as Akt and ERK1/2 (10). Immunoblotting results revealed that PPZ also decreased phosphorylation levels of Akt and ERK1/2 in HUT78 cells (Fig. 2A-D). Phosphorylation at Thr308 and Ser473 in Akt is necessary for their activation, and is regulated by PDK1 and mTORC2, respectively $(15,16)$. To reveal the effects of PPZ on upstream kinases, Ser241 phosphorylation of PDK1, an index of PDK1 activity, and Thr346 phosphorylation of NDRG1, an index of mTORC2 activity, were analyzed by immunoblotting (15,17). PPZ did not change PDK1 and NDRG1 phosphorylation levels (Fig. 2E and F), suggesting that PPZ induces Akt dephosphorylation without affecting the upstream kinases. We also investigated the effects of PPZ on upstream kinases of ERK1/2, such as B-Raf, C-Raf, and MEK1/2 $(18,19)$. PPZ decreased phosphorylation level of MEK1/2, but it did not alter B-Raf and C-Raf phosphorylation (Fig. 2G and H). These data suggested that PPZ induces MEK/ERK dephosphorylation without affecting the upstream kinases.

PPZ dephosphorylates Akt in PP2A-dependent manner and dephosphorylates ERK1/2 with no dependency on PP2A. Gutierrez et al reported that PPZ directly activates PP2A and 




B




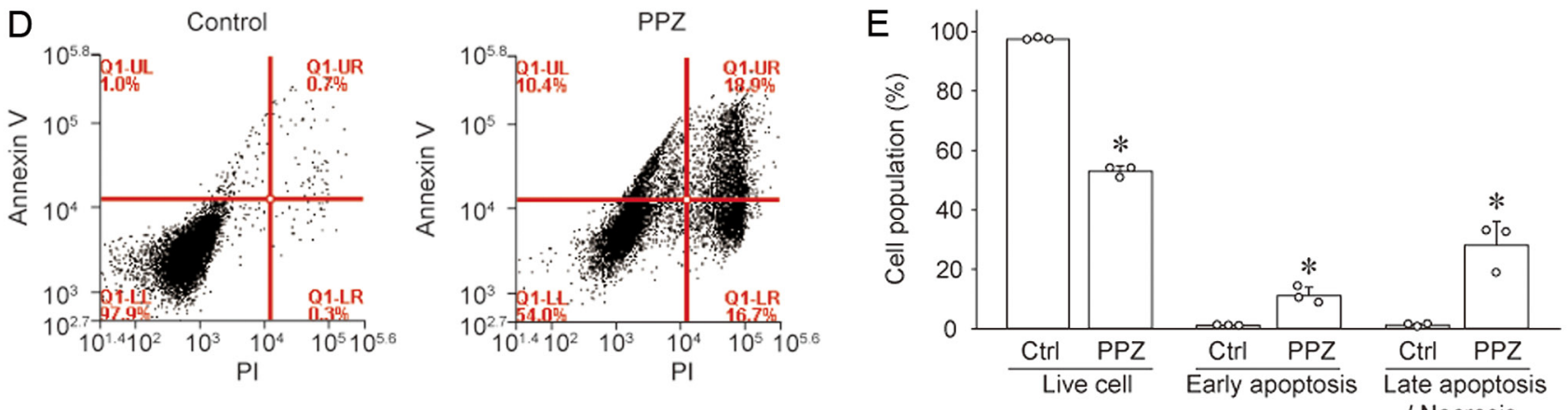

Figure 1. Perphenazine induces apoptosis in HUT78 cells. (A) The cell survival rates of HUT78, CCRF-CEM, Jurkat, and TALL1 cells treated with the indicated concentrations of PPZ were assessed. Cell survival rate was normalized to that of PPZ non-treatment and set as $100 \%$ ( $\mathrm{n}=4-6)$. Cleaved caspase 3 was detected by immunoblotting. Representative (B) images and (C) quantitative data from five independent experiments are presented. "P<0.05 vs. 0 h. HUT78 cell apoptosis was measured via flow cytometry. (D) Representative images and (E) quantitative data from three independent experiments are presented. The ratio of live cells (Annexin V-negative/PI-negative), cells in early apoptosis (Annexin V-positive/PI-negative), and in late apoptosis/necrosis (Annexin V-positive/ PI-positive) were compared between Ctrl and PPZ treatment. "P<0.005 vs. Ctrl. PPZ, perphenazine; Ctrl, control.



D

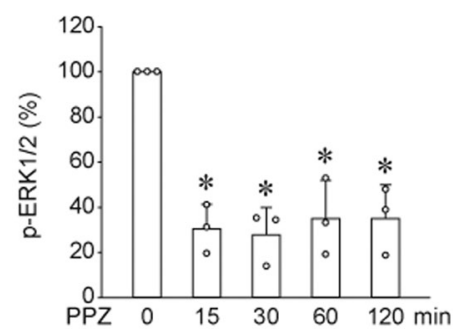

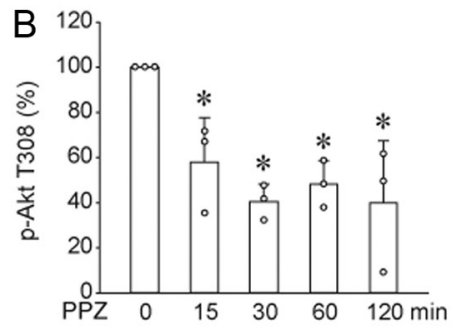

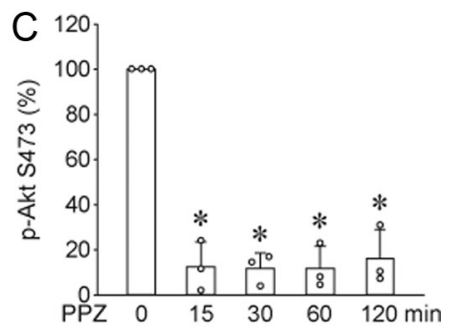

$\mathrm{E}$
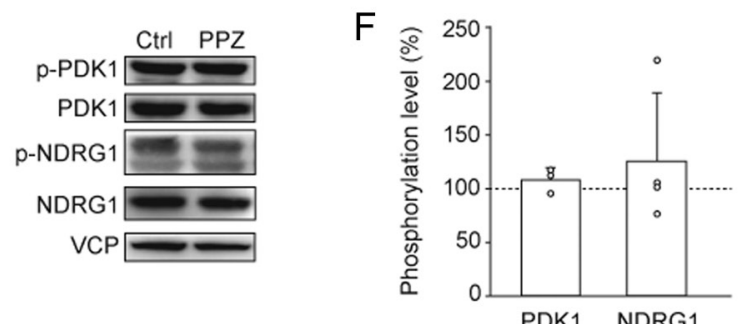
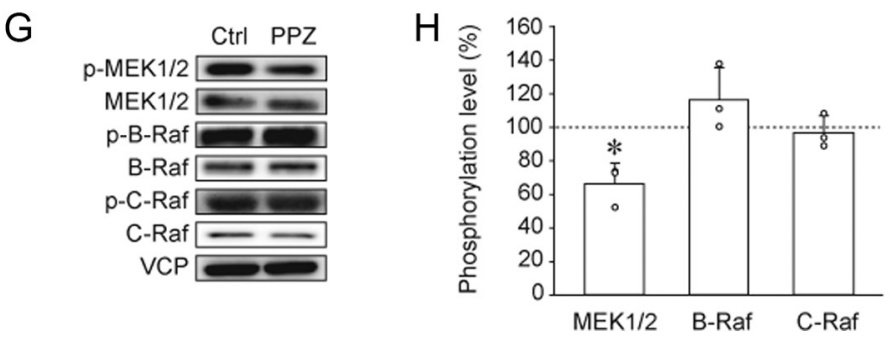

Figure 2. PPZ induces dephosphorylation of protein phosphatase $2 \mathrm{~A}$ substrates. (A, E and G) Western blotting and subsequent quantification was performed to analyze the effects of PPZ on the phosphorylation of the following proteins: (B) p-Thr308 Akt, (C) p-Ser473 Akt, (D) p-ERK1/2, (F) p-PDK1 and p-NDRG1, (H) p-MEK1/2, p-B-Raf, and p-C-Raf. Data from three to four independent experiments are presented. Ctrls were ethanol treated. "P<0.05 vs. Ctrl. or PPZ 0 min. PPZ, perphenazine; p, phosphorylated; NDRG1, N-Myc downstream-regulated gene 1; Ctrl, control; VCP, valosin-containing protein. 


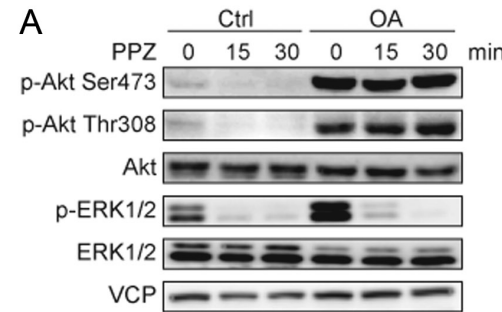

C

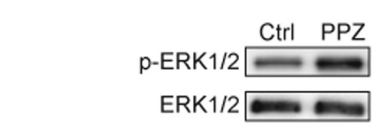

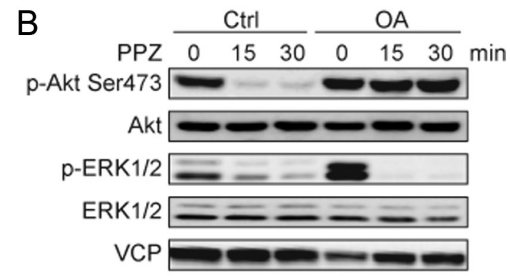

$\mathrm{D}$

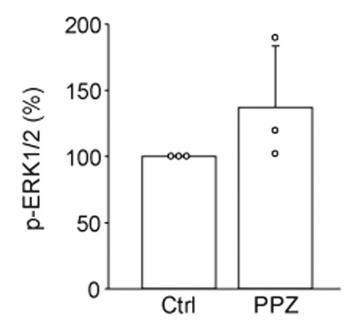

$\mathrm{E}$
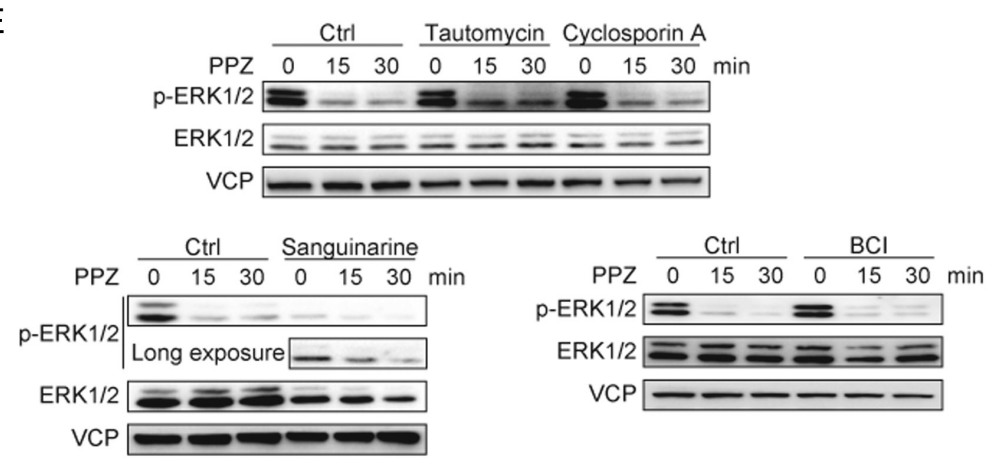

$\mathrm{F}$

G

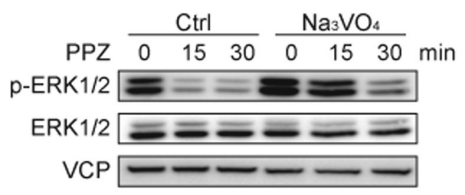

Figure 3. PPZ dephosphorylates Akt in a PP2A-dependent manner and dephosphorylates ERK1/2 with no dependency on PP2A. The phosphorylation of Akt and ERK1/2 in (A) HUT78 and (B) UL-1 cells were determined via western blotting. Representative images from three independent experiments are presented. The effect of PPZ $(10 \mu \mathrm{M})$ on the activity of recombinant MEK1/2 was analyzed by performing an in vitro kinase assay with recombinant ERK1/2 as a substrate. Phosphorylation levels of ERK1/2 were detected via (C) western blotting, with subsequent (D) quantification from three independent experiments. (E) HUT78 cells were pre-incubated with tautomycin $(1 \mu \mathrm{M})$, cyclosporin A $(10 \mu \mathrm{M})$ and sanguinarine $(10 \mu \mathrm{M})$ for $4 \mathrm{~h}$, as well as with BCI (10 $\mu \mathrm{M})$ for $1 \mathrm{~h}$. Then cells were treated with PPZ $(20 \mu \mathrm{M})$ for 15 and 30 min. The phosphorylation of ERK1/2 was detected by western blotting. Representative images from two to three independent experiments are presented. HUT78 cells, following pre-incubation with $\mathrm{Na}_{3} \mathrm{VO}_{4}(300 \mu \mathrm{M})$ for $1 \mathrm{~h}$, were treated with PPZ $(20 \mu \mathrm{M})$ for 15 and $30 \mathrm{~min}$. (F) Representative images and $(\mathrm{G})$ quantitative data from three independent experiments were presented. * $<0.05$ as indicated. PPZ, perphenazine; PP2A, protein phosphatase 2A; Ctrl, control; VCP, valosin-containing protein; p, phosphorylated; OA, okadaic acid.

induces Akt and ERK1/2 dephosphorylation, and this inhibitory effect was blocked by the PP2A inhibitor, okadaic acid (OA), and by shRNA knockdown of specific PP2A subunits (10). Thus, we tested whether OA blocks the effects of PPZ in HUT78 cells (Fig. 3A). In HUT78 cells, OA induced Akt phosphorylation, therefore, we need to adjust the exposure time. OA blocked PPZ-induced Akt dephosphorylation; however, OA did not block the dephosphorylation of ERK1/2. We previously reported that PPZ dephosphorylated Akt and ERK1/2 in the canine T-ALL cell line UL-1 (13). Similar to the effects of OA on PPZ-induced dephosphorylations in HUT78 cells, OA blocked PPZ-induced Akt dephosphorylation, but not ERK1/2 dephosphorylation in UL-1 cells (Fig. 3B). These results suggest that PPZ blocks Akt phosphorylation in a PP2A-dependent manner, however, PP2A is not involved in PPZ-induced ERK1/2 dephosphorylation. To clarify the molecular mechanism associated with these effects of PPZ, we tested whether PPZ directly inhibits MEK1/2 activity, using an in vitro kinase assay (Fig. $3 \mathrm{C}$ and D). The phosphorylation level of the MEK1/2 substrate, ERK1/2, was not blocked by PPZ, suggesting PPZ may activate some phosphatase(s) other than PP2A. Multiple phosphatases such as PP1, PP2B, PP2C, and DUSP1/6, are involved in ERK1/2 dephosphorylation (20-23). We analyzed the effects of the PP1, PP2B, PP2C, and DUSP1/6 inhibitors, tautomycin (Tau), cyclosporin A (CsA), sanguinarine, and BCI, respectively, on PPZ-induced ERK1/2 dephosphorylation (Fig. 3E). Our 

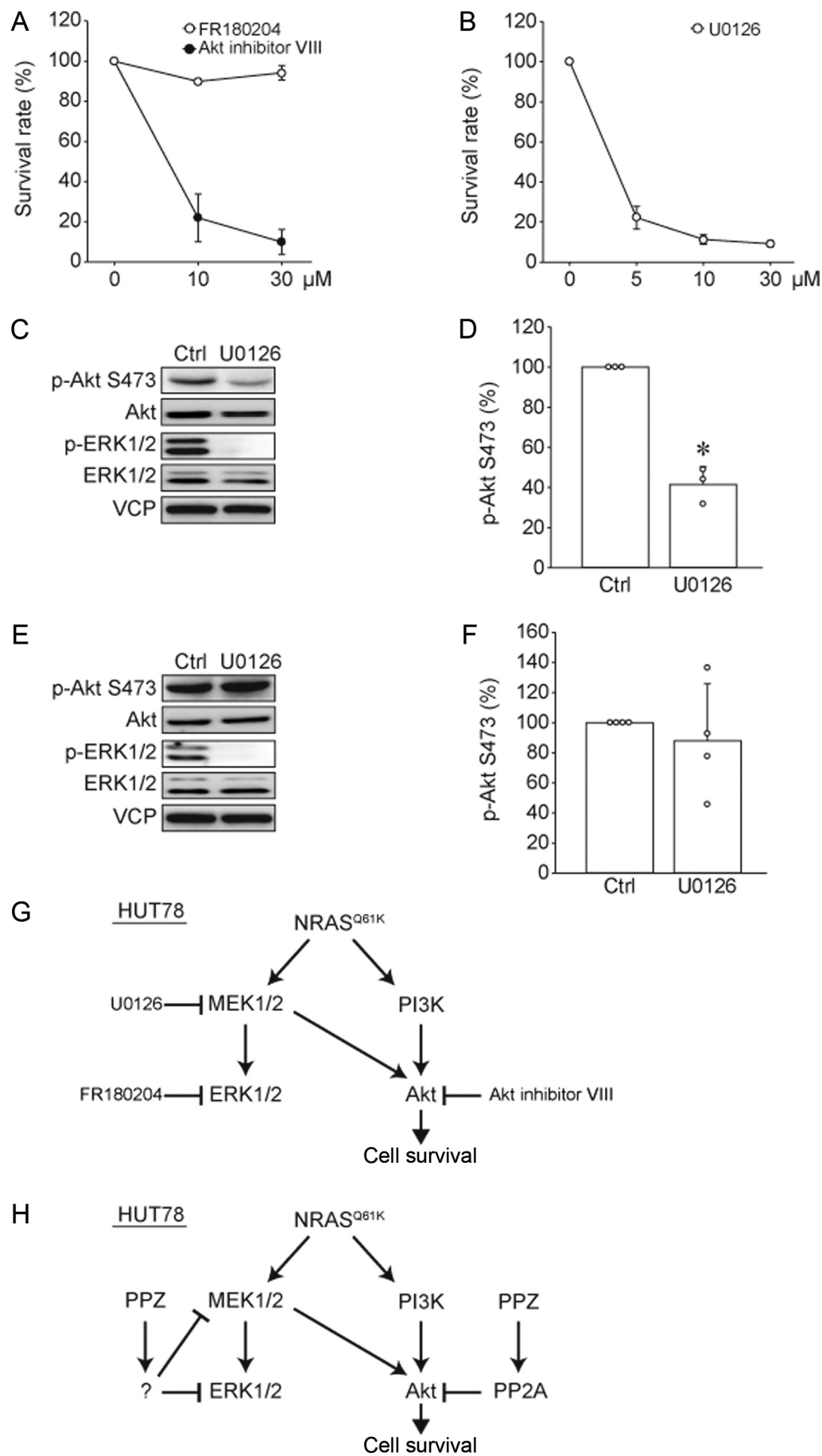

Figure 4. Akt pathway is important for the survival of HUT78 cells. (A) HUT78 cells were treated with the indicated dose of Akt inhibitor VIII (closed circle) or FR180204 (open circle) for $72 \mathrm{~h}$. Cell viability was analyzed using a CCK-8 assay. Survival rate was normalized to $0 \mu \mathrm{M}$ and marked as $100 \%$ ( $=4$ ). (B) HUT78 cells were treated with the indicated dose of U0126 for $72 \mathrm{~h}$. Cell viability was analyzed by performing a CCK8 assay. Survival rate was normalized to $0 \mu \mathrm{M}$ and marked as $100 \%(\mathrm{n}=4)$. (C and D) HUT78 and (E and F) UL-1 cells were treated with U0126 (10 $\mu \mathrm{M})$ for $24 \mathrm{~h}$. (C and E) Representative images and (D and F) quantitative data of Ser473 phosphorylation levels of Akt are presented ( $n=3-4)$. ${ }^{*} \mathrm{P}<0.05$ as indicated. (G) Cell signaling cascade of HUT78 cells demonstrating the point of action of kinase inhibitors. (H) Model of the effects of PPZ on signaling in HUT78 cells. CCK-8, cell counting kit-8; PPZ, perphenazine; PP2A, protein phosphatase 2A; NRAS, neuroblastoma rat sarcoma oncogene; Ctrl, control; p, phosphorylated; VCP, valosin-containing protein.

results show that these phosphatase inhibitors did not block PPZ-induced ERK1/2 dephosphorylation. Therefore, we tested the pan-tyrosine phosphatase inhibitor, $\mathrm{Na}_{3} \mathrm{VO}_{4}$, and observed that $\mathrm{Na}_{3} \mathrm{VO}_{4}$ slightly, but significantly, suppressed ERK1/2 dephosphorylation by PPZ (Fig. 3F and G), suggesting that a type of tyrosine phosphatase or a dual specificity phosphatase is involved in PPZ-induced ERK1/2 dephosphorylation.
Akt pathway is important for the survival of HUT78 cells. To determine which signal transduction pathway is important for the survival of HUT78 cells, Akt or ERK1/2, we analyzed the effects of an Akt inhibitor (Akt inhibitor VIII) and an ERK1/2 inhibitor (FR180204) on cell viability (Fig. 4A). Interestingly, the Akt inhibitor induced cell death in HUT78 cells in a dose-dependent manner, while the ERK1/2 inhibitor had no 
effect on cell viability. Furthermore, the MEK1/2 inhibitor, U0126, showed antitumor effects on HUT78 cells (Fig. 4B). To clarify this discrepancy, we studied the effects of this MEK1/2 inhibitor on the phosphorylation levels of Akt and ERK1/2 (Fig. 4C and D). U0126 suppressed not only ERK1/2 phosphorylation but also Akt phosphorylation, suggesting that MEK1/2 activity positively regulates Akt phosphorylation in HUT78 cells. We previously reported that UL-1 cells were sensitive to Akt inhibitor VIII, but not to FR180204 or U0126 (13). Therefore, we examined the effects of U0126 on Akt phosphorylation in UL-1 cells (Fig. 4E and F). We found that in UL-1 cells, U0126 suppressed ERK1/2 phosphorylation, but did not affect Akt phosphorylation. These results suggested that MEK inhibitor suppressed the survival of HUT78 cells by inhibiting Akt activity (Fig. 4G), and Akt inhibition is important for the antitumor effects of PPZ.

\section{Discussion}

Recently, reactivation of PP2A has attracted a lot of attention as a promising approach for cancer therapy. Small molecule activators of PP2A (SMAPs) specifically activates the PP2A B56 $\alpha$ subunit (6) and exerts anticancer effects on various cancers models such as lung cancer, breast cancer, endometrial cancer, and pancreatic neuroendocrine tumor (24-27). SMAPs were engineered from phenothiazine parent compounds supporting the activation effects of PPZ on PP2A.

Gutierrez et al reported that PPZ activates PP2A to dephosphorylate Akt and ERK1/2 in human T-ALL cell line KOPT-K1 (10). These reports were based on results from experiments using OA as well as shRNA targets in PP2A. Similarly, in the present study, we observed that OA blocked the effects of PPZ on Akt dephosphorylation; however, OA did not block PPZ-induced ERK1/2 dephosphorylation in HUT78 and UL-1 cells. One possible explanation for this discrepancy is that Gutierrez et al (10) used $1 \mu \mathrm{M}$ of OA to block ERK1/2 dephosphorylation; $1 \mu \mathrm{M}$ of $\mathrm{OA}$ is known to inhibit not only the activity of PP2A, but also that of PP1, and a lower dose, such as $100 \mathrm{nM}$, needs to be used to specifically inhibit PP2A (28). Moreover, in their results from studies using shRNA, Gutierrez et al (10) showed that PP2A knockdown effectively inhibited PPZ-induced Akt dephosphorylation, but only slightly suppressed ERK1/2 dephosphorylation. Overall, these data suggest that although PPZ dephosphorylates Akt in a PP2A-dependent manner, PP2A is not a major phosphatase in PPZ-induced ERK1/2 dephosphorylation (Fig. 4H). It has been reported that PP2A activity on ERK1/2 is regulated by the immediate early response 3 protein (IER 3 or IEX-1) (9). IER3 enhances the phosphorylation of PP2A regulatory subunit by ERK1/2 leading to suppression of PP2A activity (9). Because RAS oncogene mutation induces IER3 expression (29), and HUT78 cells express NRAS mutation (2), an increase in IER 3 protein levels may be one of the processes that could explain the lack of involvement of PP2A in PPZ-induced ERK1/2 dephosphorylation.

Which phosphatase is responsible for PPZ-induced ERK1/2 dephosphorylation? The results of our in vitro kinase assay suggest that PPZ does not directly inhibit MEK1/2 kinase activity. Inhibitors of PP1, PP2B, PP2C, and DUSP1/6 activity did not block PPZ-induced ERK1/2 dephosphorylation; however, the pan-tyrosine phosphatase inhibitor, $\mathrm{Na}_{3} \mathrm{Vo}_{4}$, partially inhibited ERK1/2 dephosphorylation, suggesting that a kind of tyrosine or dual specificity phosphatase(s) may be involved in PPZ-induced ERK1/2 dephosphorylation. The dual specificity phosphatase 4, DUSP4, dephosphorylates ERK1/2 (30). We were not able to further investigate this point owing to the unavailability of a specific DUSP4 inhibitor. A genetic screening assay using genome editing or si/shRNA will need to be conducted in future, to identify the responsible phosphatase(s).

We found that pharmacological inhibition of Akt, but not of ERK1/2, suppressed HUT78 cell growth. These results suggest that PP2A-dependent Akt dephosphorylation plays an important role in the antitumor effects of PPZ. Interestingly, a MEK inhibitor effectively suppressed HUT78 cell growth, consistent with a previous report which showed that three types of MEK inhibitors induced apoptosis in HUT78 cells (2). That study reported that HUT78 cells harbored the NRAS ${ }^{\mathrm{Q} 61 \mathrm{~K}}$ mutation that leads to enhanced MEK/ERK signaling (2). Based on our results using an ERK1/2 inhibitor, ERK1/2 activation by $\mathrm{NRAS}^{\mathrm{Q} 61 \mathrm{~K}}$ mutation is not involved in cell survival in HUT78 cell lines. We revealed that a MEK inhibitor inhibited Akt phosphorylation in HUT78 cells; however, in UL-1 cells that are resistant to MEK inhibitor, pharmacological inhibition of MEK did not affect Akt phosphorylation. These results suggest that NRAS ${ }^{\mathrm{Q} 61 \mathrm{~K}}$ mutation leads to Akt phosphorylation through MEK1/2 activation, and that Akt activity plays an important role in the survival of HUT78 cells.

As for the HUT78 cells, Akt inhibitor is enough to kill cells and PP2A activator may be not necessary to use. PP2A activators suppress multiple signals not only Akt but also such as c-myc and E2F $(31,32)$. Therefore, the effect of the PP2A activator is not limited to Akt inhibition, and the usage of Akt inhibitors and PP2A activators may be different from drug-resistant point of view. It was reported that long-term treatment with Akt inhibitor (MK-2206) induces drug resistant in pancreatic neuroendocrine tumors (PNETs). On the other hand, PNETs did not become resistant to PP2A activator even after long-term treatment (25). Overall, our data suggests that PPZ-induced Akt dephosphorylation by PP2A activation may be an attractive antitumor strategy for NRAS-mutated Sezary syndrome.

\section{Acknowledgements}

Not applicable.

\section{Funding}

This study was partially supported by Japan Society for the Promotion of Science (grant nos. 17H03915, 18J13124 and 18K05994).

\section{Availability of data and materials}

The datasets used and/or analyzed during the present study are available from the corresponding author on reasonable request.

\section{Authors' contributions}

ST and TO conceived and designed the present study, analyzed and interpreted the data, and wrote/revised the manuscript. ST 
acquired the most of the data. NK acquired the data for apoptosis and interpreted the data. TM acquired the FACS data. KS provided facilities, materials and suggestions, and interpreted the data. All authors read and approved the final manuscript.

\section{Ethics approval and consent to participate}

Not applicable.

\section{Patient consent for publication}

Not applicable.

\section{Competing interests}

The authors declare that they have no competing interests.

\section{References}

1. Foss FM and Girardi M: Mycosis fungoides and sezary syndrome. Hematol Oncol Clin North Am 31: 297-315, 2017.

2. Kiessling MK, Oberholzer PA, Mondal C, Karpova MB, Zipser MC, Lin WM, Girardi M, Macconaill LE, Kehoe SM, Hatton C, et al: High-throughput mutation profiling of CTCL samples reveals KRAS and NRAS mutations sensitizing tumors toward inhibition of the RAS/RAF/MEK signaling cascade. Blood 117: 2433-2440, 2011.

3. Vu HL and Aplin AE: Targeting mutant NRAS signaling pathways in melanoma. Pharmacol Res 107: 111-116, 2016.

4. Petit V, Raymond J, Alberti C, Pouteaux M, Gallagher SJ, Nguyen MQ, Aplin AE, Delmas V and Larue L: C57BL/6 congenic mouse NRAS ${ }^{\mathrm{Q} 61 \mathrm{~K}}$ melanoma cell lines are highly sensitive to the combination of Mek and Akt inhibitors in vitro and in vivo. Pigment Cell Melanoma Res 32: 829-841, 2019.

5. Uddin S, Hussain A, Al-Hussein K, Platanias LC and Bhatia KG: Inhibition of phosphatidylinositol 3'-kinase induces preferentially killing of PTEN-null T leukemias through AKT pathway. Biochem Biophys Res Commun 320: 932-938, 2004.

6. Leonard D, Huang W, Izadmehr S, O'Connor CM, Wiredja DD, Wang Z, Zaware N, Chen Y, Schlatzer DM, Kiselar J, et al: Selective PP2A enhancement through biased heterotrimer stabilization. Cell 181: 688-701.e16, 2020.

7. Wlodarchak N and Xing Y: PP2A as a master regulator of the cell cycle. Crit Rev Biochem Mol Biol 51: 162-184, 2016.

8. Kuo YC, Huang KY, Yang CH, Yang YS, Lee WY and Chiang CW: Regulation of phosphorylation of Thr-308 of Akt, cell proliferation, and survival by the B55alpha regulatory subunit targeting of the protein phosphatase $2 \mathrm{~A}$ holoenzyme to Akt. J Biol Chem 283: 1882-1892, 2008.

9. Letourneux C, Rocher G and Porteu F: B56-containing PP2A dephosphorylate ERK and their activity is controlled by the early gene IEX-1 and ERK. EMBO J 25: 727-738, 2006.

10. Gutierrez A, Pan L, Groen RW, Baleydier F, Kentsis A, Marineau J, Grebliunaite R, Kozakewich E, Reed C, Pflumio F, et al: Phenothiazines induce PP2A-mediated apoptosis in T cell acute lymphoblastic leukemia. J Clin Invest 124: 644-655, 2014.

11. Zonta F, Pagano MA, Trentin L, Tibaldi E, Frezzato F, Trimarco V, Facco M, Zagotto G, Pavan V, Ribaudo G, et al: Lyn sustains oncogenic signaling in chronic lymphocytic leukemia by strengthening SET-mediated inhibition of PP2A. Blood 125: $3747-3755,2015$.

12. Hartung B, Sampson S and Leucht S: Perphenazine for schizophrenia. Cochrane Database Syst Rev 2015: CD003443, 2015

13. Tsuji S, Yabe R, Usui T, Mizuno T, Ohama T and Sato K Anti-tumor effects of perphenazine on canine lymphoma. J Vet Med Sci 78: 1293-1298, 2016.

14. Ruvolo PP, Deng X, Ito T, Carr BK and May WS: Ceramide induces $\mathrm{Bcl} 2$ dephosphorylation via a mechanism involving mitochondrial PP2A. J Biol Chem 274: 20296-20300, 1999.

15. Alessi DR, James SR, Downes CP, Holmes AB, Gaffney PR, Reese CB and Cohen P: Characterization of a 3phosphoinositide-dependent protein kinase which phosphorylates and activates protein kinase Balpha. Curr Biol 7: 261-269, 1997.
16. Sarbassov DD, Guertin DA, Ali SM and Sabatini DM: Phosphorylation and regulation of Akt/PKB by the Rictor-mTOR complex. Science 307: 1098-1101, 2005.

17. García-Martínez JM and Alessi DR: mTOR complex 2 (mTORC2) controls hydrophobic motif phosphorylation and activation of serum- and glucocorticoid-induced protein kinase 1 (SGK1). Biochem J 416: 375-385, 2008.

18. Alessi DR, Saito Y, Campbell DG, Cohen P, Sithanandam G, Rapp U, Ashworth A, Marshall CJ and Cowley S: Identification of the sites in MAP kinase kinase-1 phosphorylated by p74raf-1. EMBO J 13: 1610-1619, 1994.

19. McCubrey JA, Steelman LS, Chappell WH, Abrams SL, Wong EW, Chang F, Lehmann B, Terrian DM, Milella M, Tafuri A, et al: Roles of the Raf/MEK/ERK pathway in cell growth, malignant transformation and drug resistance. Biochim Biophys Acta 1773: 1263-1284, 2007.

20. Zhou B, Wang ZX, Zhao Y, Brautigan DL and Zhang ZY: The specificity of extracellular signal-regulated kinase 2 dephosphorylation by protein phosphatases. J Biol Chem 277: 31818-31825, 2002.

21. Hu XD, Liu YN, Zhang ZY, Ma ZA, Suo ZW and Yang X: Spinophilin-targeted protein phosphatase-1 alleviated inflammatory pain by negative control of MEK/ERK signaling in spinal cord dorsal horn of rats. J Neurosci 35: 13989-14001, 2015.

22. Zhang Y, Lin DH, Wang ZJ, Jin Y, Yang B and Wang WH: $\mathrm{K}$ restriction inhibits protein phosphatase $2 \mathrm{~B}(\mathrm{PP} 2 \mathrm{~B})$ and suppression of PP2B decreases ROMK channel activity in the CCD. Am J Physiol Cell Physiol 294: C765-C773, 2008.

23. Saidak Z, Giacobbi AS, Louandre C, Sauzay C, Mammeri Y and Galmiche A: Mathematical modelling unveils the essential role of cellular phosphatases in the inhibition of RAF-MEK-ERK signalling by sorafenib in hepatocellular carcinoma cells. Cancer Lett 392: 1-8, 2017

24. Risom T, Wang X, Liang J, Zhang X, Pelz C, Campbell LG, Eng J, Chin K, Farrington C, Narla G, et al: Deregulating MYC in a model of HER2+ breast cancer mimics human intertumoral heterogeneity. J Clin Invest 130: 231-246, 2020.

25. Umesalma S, Kaemmer CA, Kohlmeyer JL, Letney B, Schab AM, Reilly JA, Sheehy RM, Hagen J, Tiwari N, Zhan F, et al: RABL6A inhibits tumor-suppressive PP2A/AKT signaling to drive pancreatic neuroendocrine tumor growth. J Clin Invest 130: 1641-1653, 2019.

26. Taylor SE, O'Connor CM, Wang Z, Shen G, Song H, Leonard D, Sangodkar J, LaVasseur C, Avril S, Waggoner S, et al: The highly recurrent PP2A A $\alpha$-subunit mutation P179R alters protein structure and impairs PP2A enzyme function to promote endometrial tumorigenesis. Cancer Res 79: 4242-4257, 2019.

27. Tohmé R, Izadmehr S, Gandhe S, Tabaro G, Vallabhaneni S, Thomas A, Vasireddi N, Dhawan NS, Ma'ayan A, Sharma N, et al: Direct activation of PP2A for the treatment of tyrosine kinase inhibitor-resistant lung adenocarcinoma. JCI Insight 4: e125693, 2019.

28. Favre B, Turowski P and Hemmings BA: Differential inhibition and posttranslational modification of protein phosphatase 1 and $2 \mathrm{~A}$ in MCF7 cells treated with calyculin-A, okadaic acid, and tautomycin. J Biol Chem 272: 13856-13863, 1997.

29. Garcia MN, Grasso D, Lopez-Millan MB, Hamidi T, Loncle C, Tomasini R, Lomberk G, Porteu F, Urrutia R and Iovanna JL: IER3 supports KRASG12D-dependent pancreatic cancer development by sustaining ERK1/2 phosphorylation. J Clin Invest 124: 4709-4722, 2014

30. Chu Y, Solski PA, Khosravi-Far R, Der CJ and Kelly K: The mitogen-activated protein kinase phosphatases PAC1, MKP-1, and MKP-2 have unique substrate specificities and reduced activity in vivo toward the ERK2 sevenmaker mutation. J Biol Chem 271: 6497-6501, 1996.

31. Farrington CC, Yuan E, Mazhar S, Izadmehr S, Hurst L, Allen-Petersen BL, Janghorban M, Chung E, Wolczanski G, Galsky M, et al: Protein phosphatase $2 \mathrm{~A}$ activation as a therapeutic strategy for managing MYC-driven cancers. J Biol Chem 295: 757-770, 2020.

32. Enjoji S, Yabe R, Tsuji S, Yoshimura K, Kawasaki H, Sakurai M, Sakai Y, Takenouchi H, Yoshino S, Hazama S, et al: Stemness is enhanced in gastric cancer by a SET/PP2A/E2F1 axis. Mol Cancer Res 16: 554-563, 2018.

This work is licensed under a Creative Commons Attribution-NonCommercial-NoDerivatives 4.0 International (CC BY-NC-ND 4.0) License. 